\title{
TEORIA UNIFICADA DA ACEITAÇÃO E USO DA TECNOLOGIA (UTAUT2): UMA VALIDAÇÃO DO MODELO A PARTIR DA IMPLANTAÇÃO DE UM ERP.
}

Raul Afonso Pommer Barbosa ${ }^{1}$

Carolina Yukari Veludo Watanabe ${ }^{2}$

Rosália Maria Passos Da Silva ${ }^{2}$

${ }^{1}$ Universidade Federal de Rondônia / UNIR

${ }^{2}$ PPGMAD / Universidade Federal de Rondônia / UNIR 


\section{TEORIA UNIFICADA DA ACEITAÇÃO E USO DA TECNOLOGIA (UTAUT2): UMA VALIDAÇÃO DO MODELO A PARTIR DA IMPLANTAÇÃO DE UM ERP.}

Resumo: Este artigo tem como principal objetivo, procurar evidências de validade do instrumento de mensuração da Teoria Unificada da Aceitação e Uso da Tecnologia-UTAUT2. Foram entrevistados 60 funcionários de uma empresa que passou pelo processo de implantação de um ERP recentemente. Os resultados apontam para evidências de validade convergente e descriminante. Este artigo contribui para a Teoria Unificada da Aceitação e Uso da Tecnologia (UTAUT2) na medida, que, é aplicada em diferentes ambientes, evidenciando características que possam permitir sua generalização. Por fim, no âmbito prático, é possível utilizar esse instrumento para avaliar e planejar a aceitação de uma nova tecnologia no âmbito organizacional.

Palavras-chave: Intenção de uso de sistemas de informação. Enterprise Resource Planning (ERP). UTAUT 2.

\section{Introdução}

O UTAUT foi modelo desenvolvido para ser um ponto de partida para investigar a adoção de TI e pode ser usado para identificar os fatores que influenciam a intenção de usá-la, bem como para ser adotada por uma organização. A Teoria Unificada de Aceitação e Uso da Tecnologia - UTAUT (Unified Theory of Acceptance and Use of Technology), atualmente é considerado o modelo mais completo, uma vez que abrange outros oito estudos sobre aceitação de tecnologia e os unifica em um único modelo (SILVA, 2015) e centraliza os fatores determinantes em críticos e em contingências relacionadas com a previsão da intenção comportamental de usar uma tecnologia e uso de tecnologia, principalmente em contextos organizacionais (VENKATESH et al., 2003). Novas variáveis incluídas no UTAUT 2 (Motivação Hedônica, Preço e Hábito) trouxeram novas perspectivas para o entendimento da aceitação e do uso de tecnologia por parte dos indivíduos no contexto do consumo. Venkatesh et al, (2012), cita como limitações do estudo, a necessidade de que o modelo seja testado em outros lugares para fins de generalização de seus resultados, visto que a pesquisa foi realizada dentro das especificidades socioeconômicas e culturais de Hong Kong. No contexto brasileiro ainda há poucos estudos publicados sobre o UTAUT2. Desta forma este artigo busca validar o modelo da Teoria Unificada da Aceitação e Uso de Tecnologia (UTAUT2) a partir da aplicação do questionário em funcionários de uma empresa que passou pelo processo de implantação de um ERP.

O restante do artigo está organizado da seguinte maneira: Referencial teórico onde são discutidos os conceitos e teorias estudadas, metodologia aplicada para validar a escala de mensuração, análise de resultados e a conclusão sobre o problema de pesquisa deste artigo.

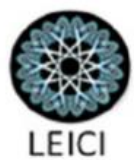




\section{Referencial Teórico}

\subsection{UTAUT}

O UTAUT é um modelo unificado que integra elementos de diversos modelos, como TRA, TAM e TAM2. Oito construtos são fundamentais para determinar a intenção de uso efetivo em sistemas de TI. Quatro desses construtos são determinantes e influenciam diretamente na aceitação da tecnologia e o comportamento de uso: Expectativa de Desempenho, Expectativa de Esforço, Influência Social e Condições Facilitadoras. Outros quatro construtos são fatores moderadores e não influenciam diretamente à aceitação da tecnologia e o comportamento de uso: Gênero, Idade, Experiência e Voluntariedade. Centraliza os fatores determinantes em críticos e em contingências relacionadas com a previsão da intenção comportamental de usar uma tecnologia e uso de tecnologia, principalmente em contextos organizacionais (VENKATESH et al., 2003). A intenção comportamental refere-se ao grau em que uma pessoa (usuário) formulou planos conscientes para realizar ou não algum comportamento futuro uso especificado. A intenção comportamental refere-se ao grau em que uma pessoa (usuário) formulou planos conscientes para realizar ou não algum comportamento futuro uso especificado.

A expectativa de desempenho é definida como o grau em que um indivíduo acredita que a utilização do sistema irá ajudá-lo a conseguir ganhos de desempenho no trabalho. É composto por cinco constructos: utilidade percebida, motivação extrínseca, ajuste a tarefa, vantagem relativa e expectativas de resultados. A expectativa de desempenho foi construída por Venkatesh (2003, p. 447) a partir de cinco constructos já existentes: utilidade percebida (DAVIS, 1989; DAVIS et al., 1989), motivação mextrínseca (THOMPSON et al., 1991), vantagem relativa (MOORE;BENBASAT,1996) e expectativa de resultado (COMPEAU; HIGGINS, 1995, JOHNSON; MARAKAS, 2000).

O segundo fator considerado no modelo é a expectativa de esforço, o qual Venkatesh et al. (2003) definiram como o grau de facilidade associada ao uso do sistema. Tal construto da UTAUT se originou a partir de três construtos de diferentes teorias: a) Facilidade de uso percebida do modelo TAM e TAM2 (DAVIS, 1989; DAVIS et al., 1989); b) Complexidade do modelo MPCU (THOMPSON et al., 1991); e c) Facilidade de uso do modelo IDT (MOORE; BENBASAT, 1991). Expectativa de esforço é definida como o grau de facilidade associado com o uso do sistema. Compreende três constructos: facilidade de utilização percebida, complexidade e a facilidade de utilização. É possível considerar que expectativa de esforço seja definida como o grau de facilidade associada com a utilização do sistema, sendo referente à quantidade de esforço percebido que o usuário precisa colocar para aprender e operar o mesmo.

A influência social refere-se ao grau em que um indivíduo percebe que outras pessoas importantes acreditam que ele deveria usar o sistema. Os principais constructos de influência social são: norma subjetiva, fatores sociais e imagem. Formulada por Venkatesh et al. (2003) a partir de três construtos: a) Norma subjetiva dos modelos TRA, TAM2, TPB, DTPB (DAVIS et al., 1989; TAYLOR; TODD, 1995); b) Fatores sociais do modelo MPCU (THOMPSON et al., 1991); e c) Imagem do modelo IDT (MOORE; BENBASAT, 1991).

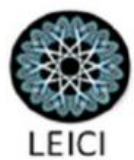


Condições Facilitadoras, é o grau em que um indivíduo acredita que exista uma infraestrutura organizacional e técnica suficiente para suportar a utilização do sistema. Esta definição é apoiada por três constructos diferentes: controle comportamental percebido, condições e compatibilidade. Esse fator é formado da mesma forma que os anteriores a partir de três construtos: a) Controle percebido do comportamento do modelo DTPB (TAYLOR; TODD, 1995); b) Condições facilitadoras do modelo MPCU (THOMPSON et al., 1991); e c) Compatibilidade do modelo IDT (MOORE; BENBASAT, 1991). Todos esses construtos podem ser observados de maneira resumida no Quadro 1 abaixo.

\begin{tabular}{|c|c|c|}
\hline Construto & Definiçăo & Origem \\
\hline $\begin{array}{l}\text { Expectativa } \\
\text { desempenho }\end{array}$ & $\begin{array}{l}\text { "Grau em que um individuo } \\
\text { acredita que o uso do sistema vai } \\
\text { ajudá-lo a atingir ganhos no } \\
\text { resultado do traballo". }\end{array}$ & $\begin{array}{l}\text { - Utilidade Percebida (TAM/TAM2 e } \\
\text { DTPB) } \\
\text { - Motivação extrinseca (MM) } \\
\text { - Adequação da função (MPCU) } \\
\text { - Vantagem relativa (IDT) } \\
\text { - Expectativa de resultados (SCT) } \\
\end{array}$ \\
\hline Expectativa de esforço & $\begin{array}{l}\text { "Grau de facilidade associada ao } \\
\text { uso do sistema" }\end{array}$ & $\begin{array}{l}\text { - Facilidade de uso percebida } \\
\text { (TAM/TAM2) } \\
\text { - Complexidade (MPCU) } \\
\text { - Facilidade de uso (IDT) }\end{array}$ \\
\hline Influẻncia social & $\begin{array}{l}\text { "Grau em que um individuo } \\
\text { percebe que outras pessoas } \\
\text { importantes acreditam que ele } \\
\text { deveria usar o novo sistema". }\end{array}$ & $\begin{array}{l}\text { - Norma subjetiva (TRA, TAM2, TPB, } \\
\text { DTPB) } \\
\text { - Fatores sociais (MPCU) } \\
\text { - Imagem (IDT) }\end{array}$ \\
\hline $\begin{array}{l}\text { Condiçōes } \\
\text { facilitadoras }\end{array}$ & $\begin{array}{l}\text { "Grau em que um individuo } \\
\text { acredita que existe uma } \\
\text { infraestrutura organizacional e } \\
\text { técnica para suportar o uso do } \\
\text { sistema". }\end{array}$ & $\begin{array}{l}\text { - Controle percebido do } \\
\text { comportamento (DTPB) } \\
\text { - Condiçōes facilitadoras (MPCU) } \\
\text { - Compatibilidade (IDT) }\end{array}$ \\
\hline
\end{tabular}

Fonte: Venkatesh et al., 2003.

O modelo UTAUT ainda prevê ainda quatro constructos moderadores da intenção e uso da TI: o gênero, a idade, a experiência do indivíduo e a voluntariedade (SILVA, 2015), (VENKATESH et al., 2003). A experiência refere-se ao grau de familiaridade do usuário com o sistema em questão, familiaridade esta adquirida por meio do uso prévio da tecnologia; e a voluntariedade contempla o grau em que o usuário acredita ser obrigatório ou não o uso do sistema em seu trabalho (SILVA, 2015) (VENKATESH et al., 2003). Gênero, idade, experiência e voluntariedade de uso são fatores que podem afetar os determinantes diretos da intenção comportamental e do comportamento de uso (expectativa de desempenho, expectativa de esforço, influência social e condições facilitadoras) (SILVA, 2015) (VENKATESH et al., 2003). A inter relação desses construtos pode ser melhor visualizada a partir da Figura 1. 
Figura 1: Modelo Conceitual do UTAUT.

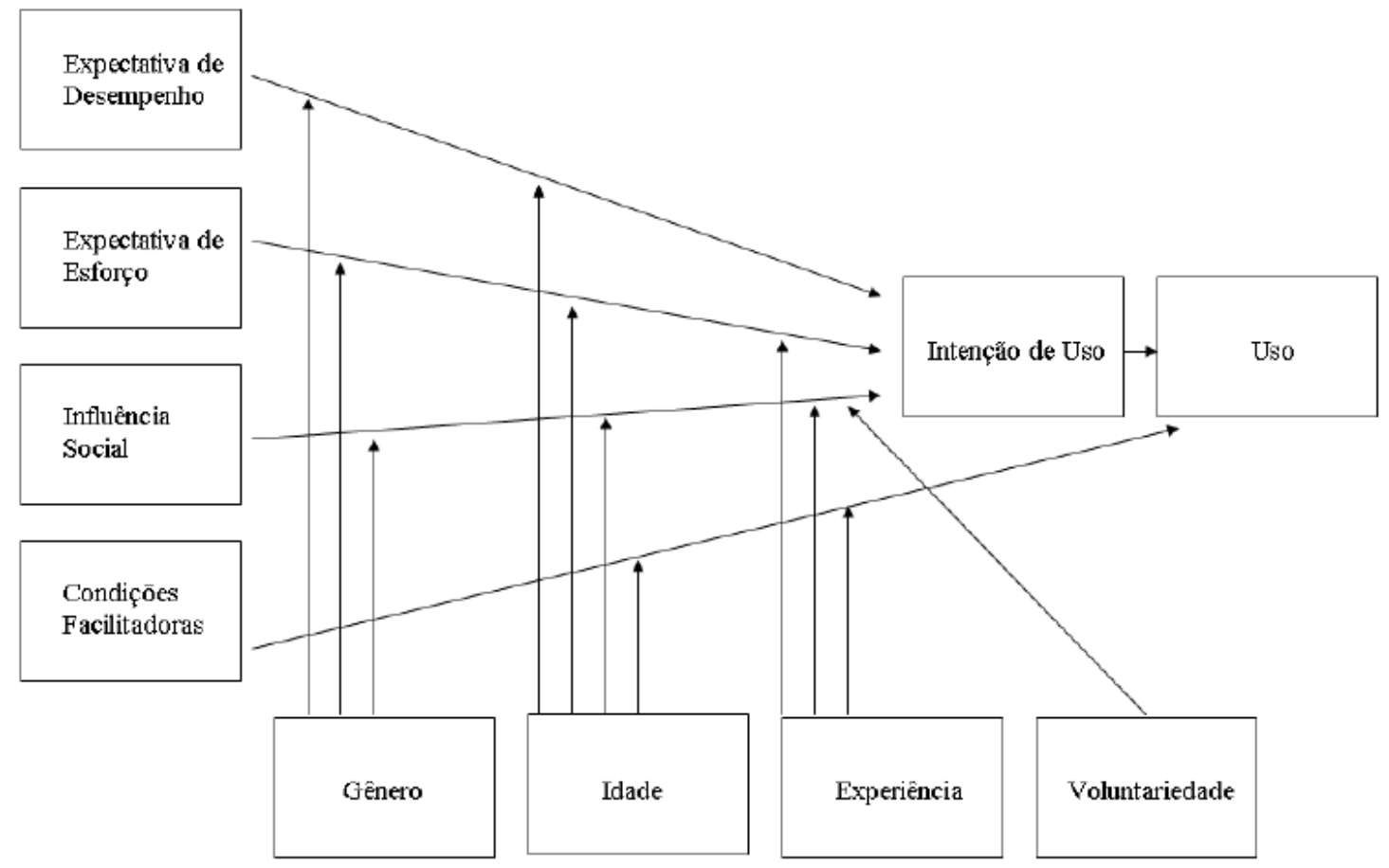

Fonte: Venkatesh e Morris. (2003).

\subsection{UTAUT 2}

O UTAUT 2 foi elaborado para verificar a aceitação e o uso de tecnologia no contexto do consumo. Utilizou um modelo estrutural, em que as variáveis, Expectativa de desempenho, Expectativa de Esforço, Influência Social, Condições Facilitadoras, Motivações Hedônicas, Preço e Hábito buscavam explicar o comportamento da variável Intenção de Comportamento, como também as variáveis Condições Facilitadoras e Intenção de Comportamento buscavam explicar a variável Intenção de Uso. Além disso, apresentou como variáveis moderadoras: gênero, idade e experiência. O modelo explicou $74 \%$ da variância da Intenção de Comportamento e 52\% para comportamento de uso, sendo considerado eficaz para predizer a aceitação e uso de tecnologia no contexto do consumo. A UTAUT enfatiza a importância do valor utilitário (motivação extrínseca). A construção ligada à utilidade, o saber, a expectativa de desempenho, tem sido consistentemente mostrada como o preditores mais fortes da intenção comportamental (VENKATESH et al., 2003). Complementar a essa perspectiva da teoria da motivação é a motivação intrínseca ou hedônica (VALLERAND, 1997). A motivação hedônica foi incluída como um preditor em muitas pesquisas sobre o comportamento do consumidor (HOLBROOK e HIRSCHMAN, 1982) e pesquisas anteriores de Sistemas de Informação no contexto do uso da tecnologia do consumidor (BROWN e VENKATESH, 2005). No constructo da expectativa de esforço, nas configurações organizacionais, os funcionários avaliam o tempo e o esforço na formação de pontos de vista sobre o esforço geral associado com a aceitação e uso de tecnologias. Em um contexto de uso 
da tecnologia de consumo, o preço também é um fator importante, pois, ao contrário das tecnologias do local de trabalho, os consumidores devem suportar os custos associados à compra de dispositivos e serviços. Consistente com este argumento, muitas pesquisas de comportamento do consumidor incluíram construções relacionadas ao custo para explicar as ações dos consumidores (DODDS et al., 1991).

Finalmente, UTAUT e modelos relacionados dependem da intencionalidade como um mecanismo teórico subjacente fundamental que impulsiona o comportamento. Muitos, incluindo detratores desta classe de modelos, argumentaram que a inclusão de mecanismos teóricos adicionais é importante. Em um uso, em vez de aceitação inicial, o hábito do contexto mostrou ser um fator crítico que prevê o uso da tecnologia (KIM e MALHOTRA, 2005; KIM et al., 2005; LIMAYEM et al., 2007). Com base nas lacunas acima mencionadas na UTAUT e na explicação teórica associada fornecida, foi adicionado a motivação hedônica, preço e hábito em UTAUT para adequá-la ao contexto de uso da tecnologia do consumidor.

Figura 2: Modelo Conceitual do UTAUT 2.

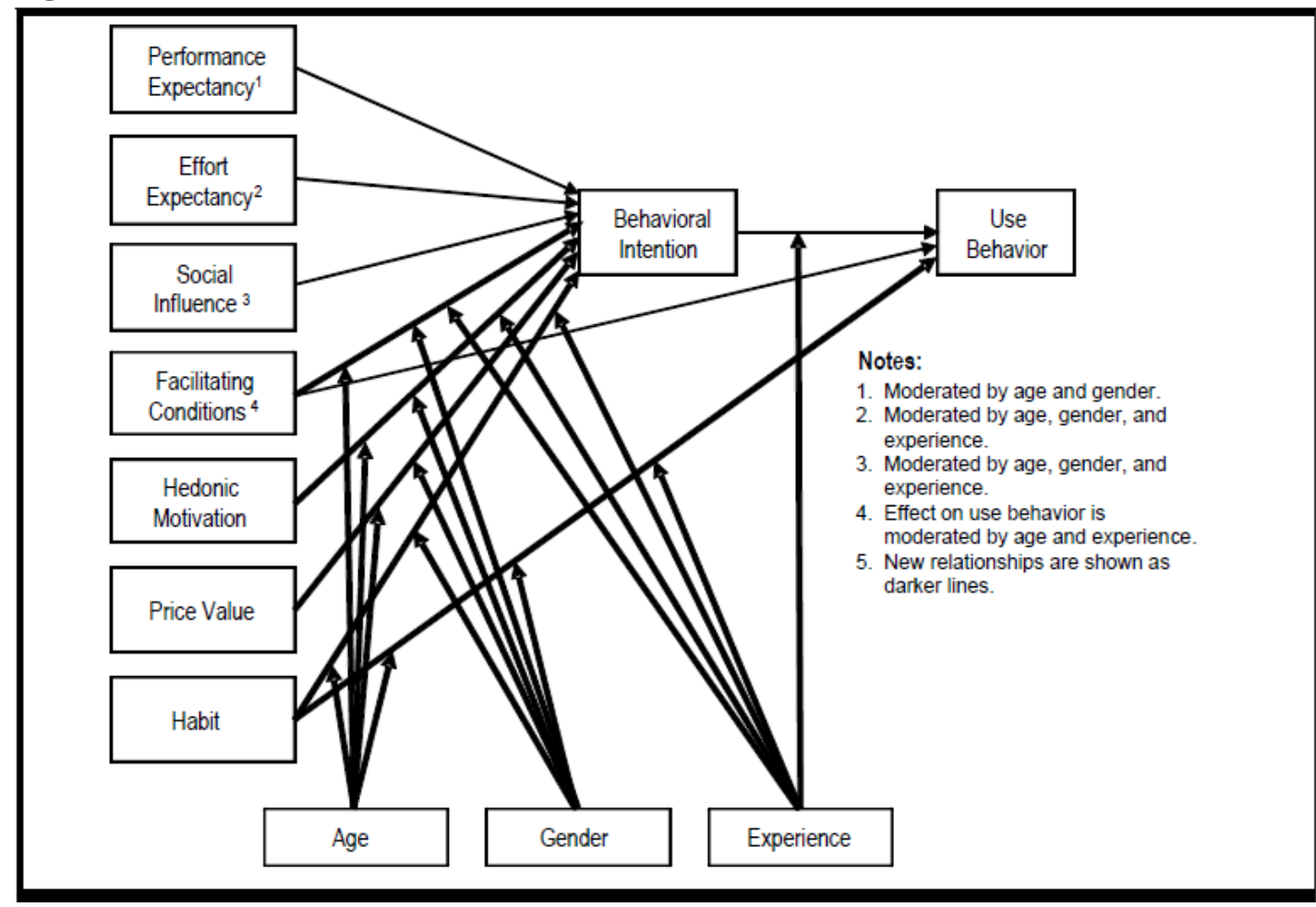

Fonte: Venkatesh, Thong e Xu (2012).

\section{Metodologia}

Nesta sessão é descrito o percurso metodológico, bem como o fundamento teórico utilizado neste artigo. A sessão está estruturada na sequência dos passos seguidos para validar a escala de aceitação e do uso de tecnologia por parte dos funcionários em uma empresa que implantou um ERP. 
O questionário utilizado neste artigo é um adaptação do questionário de Venkatesh et al (2012). A amostra foi composta por 60 funcionários da empresa estudada, que responderam o questionário de forma voluntaria. De acordo com o teorema central do limite, em experimentos utilizando seres humanos, uma amostra de 15 indivíduos por grupo pode ser considerada suficiente, desde que o desvio padrão das amostras não seja muito diferente e que elas tenham distribuição simétrica. Entretanto, com 30 sujeitos em cada grupo, não há necessidade de levar em consideração a distribuição da amostra, pois quando o tamanho da amostra aumenta, a distribuição amostral da sua média tende a aproximar-se de uma distribuição normal.

Para checar a confiabilidade da escala e verificar a correlação entres os itens do construto, foi utilizado o método mais aceito entre os pesquisadores, ou seja, fazendo uso do coeficiente alpha de Cronbach com auxílio do software SPSS versão 21. A confiabilidade pode ser definida em o quanto a escala é constante em seus resultados, consistindo em analisar a ausência de erros aleatórios presentes na mesma. $\mathrm{O}$ alpha de Cronbach mede o percentual de variação total da escala (somada) que é ditada por uma variação do conjunto de pares de covariâncias entre as variáveis. Desta forma, o coeficiente alpha é calculado da variância das respostas individuais e da variância da somatória dos componentes do questionário. Os valores de alpha podem variar de 0 a 1 , sendo que quanto mais próximo de 1 , maior será a evidência de consistência interna, e maior a indicação de confiabilidade do conjunto de itens da escala (COSTA, 2011).

Foi realizada uma análise fatorial confirmatória (AFC), que consiste em um procedimento de redução de variáveis, a partir da agregação de um determinado conjunto de itens. Predefinimos a estrutura fatorial e testamos a hipótese de aderência do conjunto de itens aos fatores, assim, verificando indícios de validade de constructo convergente e descriminante.

A validade de construto visa verificar o comportamento da medida em relação a uma expectativa teórica associada à definição do construto. A validade da escala pode ser definida pelo grau que um instrumento de medida realmente mede o que está se propondo a medir. Tendo em mente a afirmação, considerou-se que para fundamentar a validade da escala, indispensável para qualquer instrumento de medida - foi necessário analisar a validade da proposta de mensuração; o fato de a medida ter alto nível de confiança não significa que seja uma medida válida, mas uma medida válida é necessariamente confiável (COSTA, 2011).

\subsection{Análise dos Resultados}

Nesta sessão, serão analisados os resultados obtidos. A análise da confiabilidade para o construto "Expectativa de Performance", demonstrou um alpha de 0.847; O construto "Expectativa de Esforço" demonstrou um alpha de 0.858; O construto "Influência Social" demonstrou um alpha de 0.818 , O construto "Intenção de Uso" demonstrou um alpha de 0.815 e o constructo "Motivação Hedônica" demonstrou um alpha de 0.862 . Desta maneira, indicaram uma confiabilidade ótima e não sendo necessário excluir algum item para aumentar a confiabilidade desses construtos (COSTA, 2011).
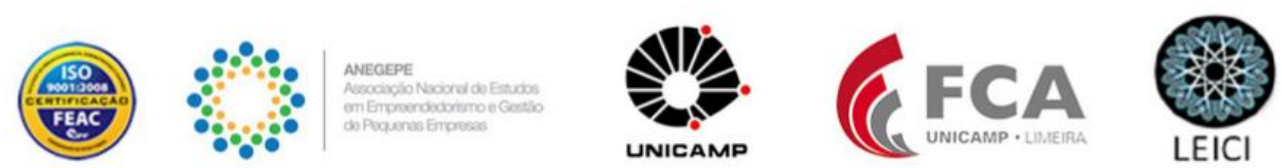
O construto "Condições Facilitadoras" demonstrou um alpha de 0.746, considerado uma confiabilidade boa (COSTA, 2011). Não sendo necessário excluir algum item para aumentar a confiabilidade.

O construto "Preço" demonstrou um alpha de 0.502, considerado uma confiabilidade não aceitavel (COSTA, 2011). Foi necessário excluir o item "PV1" para aumentar a confiabilidade. Após a exclusão do item "PV1", o Alpha de Cronbach foi para 0.663, considerado uma confiabilidade regular (COSTA,2011).

O construto "Hábito" demonstrou um alpha de 0.601 , considerado uma confiabilidade regular (COSTA, 2011). Optou-se por excluir o item "HT2" para aumentar a confiabilidade. Após a exclusão do item "HT2", o Alpha de Cronbach para o construto "Hábito" foi para 0.814, considerado uma confiabilidade ótima (COSTA,2011).

\subsection{Validade de Construto e Análise Fatorial}

Foram utilizados alguns subtipos da validade de construto neste artigo. O primeiro deles, denominado validade convergente, possui três alternativas para definí-lo: a primeira é a associação da validade convergente ao grau em que os indicadores designados para medir o mesmo construto são relacionados e convergentes; a segunda é que há a possibilidade de considerar a existência de uma validade convergente quando duas medidas distintas de um mesmo construto confirmam a expectativa de serem fortemente relacionadas, caso a correlação seja elevada entre duas medidas, sem a suposição que a escalas sejam formativas ou refletivas; e uma terceira em que há convergência entre diferentes métodos usados para medir um mesmo construto, atentando-se para o fato de que não são medidas diferentes, mas sim métodos diferentes e não havendo também a suposição de que as escalas sejam formativas ou refletivas (COSTA, 2011). O segundo subtipo de validade de construto é a validade divergente ou discriminante, que se refere ao grau em que duas medidas, designadas para medir construtos distintos, são realmente diferentes, ou seja, validade pela qual se certifica que as medidas de diferentes construtos não se comportam como se estivessem mensurando o mesmo construto.

Para verificar a valida convergente, a forma de verificação foi avaliar os pares de correlação entre os indicadores ou pela análise fatorial. Para analisar escalas de múltiplos itens é preciso verificar se as cargas fatoriais são consistentemente ligadas ao fator associado à escala e conferir a aderência do item ao fator. Para verificar a aderência, foram observadas as saídas do processo de extração de fatores nos de procedimentos de Análise Fatorial Confirmatória (AFC) com o auxilo do software SPSS versão 21, analisando inicialmente as cargas fatoriais dos múltiplos itens. Foi analisado se as cargas fatoriais foram maiores do que 0,4 (de preferência 0,6), considerado uma boa aderência dos itens aos fatores. Para avaliar a validade discriminante, foi verificado se os itens do construto tem baixas cargas fatoriais quando comparadas com os demais construtos de um instrumento de mensuração, caso esse grau seja baixo ou nulo, teremos evidências de validade discriminante (COSTA, 2011).

Para o fator "Hábito" (PE), os itens demonstraram as seguintes cargas fatoriais: "O uso do sistema de ERP tornou-se um hábito para mim" com carga fatorial de 0,728, "Eu devo usar o sistema de ERP" com carga fatorial de 0,775, e "Usar o sistema de ERP tornou-se natural para mim" com carga fatorial de 0,803 . Havendo evidências de validade convergente dos itens

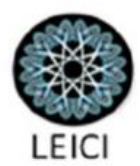


para o construto "Hábito". Quando comparado com os demais fatores, os itens apresentaram cargas fatoriais baixas, indicando validade discriminante para o construto "Hábito".

Para o fator "Expectativa de Performance" (PE), os itens demonstraram as seguintes cargas fatoriais: "Eu acho o sistema de ERP útil na minha vida diária" com carga fatorial de 0,599, "Usar o sistema de ERP aumenta minhas chances de alcançar as coisas que são importantes para mim" com carga fatorial de 0,805, "Usar o sistema de ERP me ajuda a realizar as coisas mais rapidamente" com carga fatorial de 0,818 , "Usar o sistema aumenta minha produtividade" com carga fatorial de 0,703. Havendo evidências de validade convergente dos itens para o construto "Expectativa de Performance". Quando comparado com os demais fatores, os itens apresentaram cargas fatoriais baixas, indicando validade discriminante para o construto "Expectativa de Performance".

Para o fator "Expectativa de Esforço" (EE), os itens demonstraram as seguintes cargas fatoriais: "Aprender a usar o sistema de ERP é fácil para mim" com carga fatorial de 0,808, "Minha interação com o sistema de ERP é clara e compreensível" com carga fatorial de 0,822, "O sistema de ERP é fácil de usar" com carga fatorial de 0,878 , "É fácil, para mim, ser habilidoso no uso do sistema de ERP" com carga fatorial de 0,604. Havendo evidências de validade convergente dos itens para o construto "Expectativa de Esforço". Quando comparado com os demais fatores, os itens apresentaram cargas fatoriais baixas, indicando validade discriminante para o construto "Expectativa de Esforço".

Para o fator "Motivação Hedônica" (HM), os itens demonstraram as seguintes cargas fatoriais: "Usar o sistema de ERP é legal" com carga fatorial de 0,648, "Usar o sistema de ERP é agradável" com carga fatorial de 0,850, "Usar o sistema de ERP é muito divertido" com carga fatorial de 0,766. Havendo evidências de validade convergente dos itens para o construto "Motivação Hedônica". Quando comparado com os demais fatores, os itens apresentaram cargas fatoriais baixas, indicando validade discriminante para o construto "Motivação Hedônica".

Para o fator "Influência Social" (SI), os itens demonstraram as seguintes cargas fatoriais: "Pessoas que são importantes para mim pensam que eu deveria usar o sistema de ERP" com carga fatorial de 0,800 , "As pessoas que influenciam meu comportamento pensam que eu deveria usar o sistema de ERP" com carga fatorial de 0,887, "Pessoas cujas opiniões que eu valorizo preferem usar o sistema de ERP" com carga fatorial de 0,615. Havendo evidências de validade convergente dos itens para o construto "Influência Social". Quando comparado com os demais fatores, os itens apresentaram cargas fatoriais baixas, indicando validade discriminante para o construto "Influência Social".

Para o fator "Condições Facilitadoras" (FC), os itens demonstraram as seguintes cargas fatoriais: "Tenho os recursos necessários para usar o sistema de ERP" com carga fatorial de 0,809, "Tenho o conhecimento necessário para usar o sistema de ERP" com carga fatorial de 0,704 , "O sistema de ERP é compatível com outras tecnologias que uso" com carga fatorial de 0,558, "Posso obter ajuda de outros quando tenho dificuldades em usar o sistema de ERP" com carga fatorial de 0,320. Houve evidências de validade convergente para o construto "Condições Facilitadoras" apenas os itens 'FC1', ' 'FC2" e "FC3", o item "Posso obter ajuda de outros quando tenho dificuldades em usar o sistema de ERP" (FC4) obteve baixa carga fatorial. Quando comparado com os demais fatores, apenas os itens "FC1" e "FC2" apresentaram cargas fatoriais baixas, indicando validade discriminante para o construto

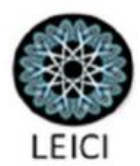


"Condições Facilitadoras". O item "O sistema de ERP é compatível com outras tecnologias que uso" (FC3) apresentou uma carga fatorial média para o fator "Condições Facilitadoras".

Para o fator "Preço" (PV), os itens demonstraram as seguintes cargas fatoriais: "O sistema de ERP tem um bom custo-benefício" com carga fatorial de 0,335 e "Ao preço atual, o sistema de ERP oferece um bom retorno" com carga fatorial de 0,818. Havendo evidências de validade convergente apenas o item "Ao preço atual, o sistema de ERP oferece um bom retorno" para o construto "Preço". Quando comparado com os demais fatores, os itens apresentaram cargas fatoriais baixas, indicando validade discriminante para o construto "Preço".

Por fim, para o fator "Intenção de Uso" (BI), os itens demonstraram as seguintes cargas fatoriais: "Pretendo continuar usando o sistema de ERP no futuro" com carga fatorial de 0,190, "Eu sempre tentarei usar o sistema de ERP na minha vida diária" com carga fatorial de 0,552, "Planejo continuar a usar o sistema de ERP com frequência" com carga fatorial de 0,449 . Havendo evidências de validade convergente e discriminante apenas o item "Eu sempre tentarei usar o sistema de ERP na minha vida diária " para o construto "Intenção de Uso". Quando comparado com os demais fatores, os itens "Pretendo continuar usando o sistema de ERP no futuro" e "Planejo continuar a usar o sistema de ERP com frequência" apresentaram cargas fatoriais 0.641 e 0.546 para o fator "Hábito" respectivamente, indicando uma forte aderência, e desta maneira, devem ser remodelados ou excluídos do instrumento de mensuração.

\section{Conclusão e Limitações}

O principal objetivo deste artigo foi procurar evidências de validade do instrumento de mensuração da Teoria Unificada da Aceitação e Uso da Tecnologia-UTAUT2. Os itens de cada fator, de forma geral, demonstraram resultados considerados satisfatórios. No entanto, é recomendada a elaboração de novos itens para os fatores "Condições Facilitadoras" e "Intenção de Uso" e novas validações da escala da teoria UTAUT2, dado que os construtos podem variar de acordo com o ambiente pesquisado e pela variação do tempo. Os significados que os construtos têm em determinados momentos podem mudar com a passagem dos anos, devido à quebra de paradigmas e mudanças comportamentais das sociedades.

Dos 29 itens inicias, 2 itens foram excluídos com a finalidade de aumentar a confiabilidade com o cálculo do Alpha de Cronbach. Para o fator "Preço" foi excluído o item "O sistema de ERP é barato" (PV1) e para o fator "Hábito" foi excluído o item "Sou viciado em usar o sistema de ERP" (HT2). Por fim, esse artigo apresentou validade convergente e discriminante através da Análise Fatorial Confirmatória, demonstrando aderência dos itens a escala de mensuração pela análise das cargas fatoriais.

Para futuros estudos é proposto o uso da modelagem de equação estrutural para avaliar a confiabilidade e validade da escala de mensuração da teoria UTAUT2 e a construção de novos itens, correlacionando-os com os construtos de caráter formativos e de controle, sendo esta, uma limitação deste artigo.

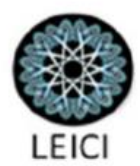




\section{Referências}

BROWN, S. A., and VENKATESH, V. 2005. "Model of Adoption of Technology in the Household: A Baseline Model Test and Extension Incorporating Household Life Cycle," MIS Quarterly (29:4), pp. 399. 426.

COMPEAU, D. R., and HIGGINS, C. A. "Application of Social Cognitive Theory to Training for Computer Skills,” Information Systems Research (6:2), 1995a, pp. 118-143.

COMPEAUS, D. R., and HIGGINS, C. A. "Computer Self-Efficacy: Development of a Measure and Initial Test,” MIS Quarterly (19:2), 1995b, pp. 189-211.

COSTA, Francisco José da. Mensuração e Desenvolvimento de Escalas: Aplicações em Administração. Rio de Janeiro: Editora Ciência Moderna Ltda, 2011.

Dissertação de Mestrado, Descrição: Aplicação do Modelo UTAUT na Avaliação da Intenção de Uso de Sistemas ERP. , Situação: Concluído; Natureza: Pesquisa. , Integrantes: Jorge Marcelino Bassalo da Silva Coordenador.

DA SILVA, J. M. B. Aplicação do modelo UTAUT na avaliação da intenção de uso de sistemas ERP. 2009, 91f. Dissertação (Mestrado Profissionalizante em Administração). Programa de Pós-Graduação e Pesquisa em Administração e Economia. Faculdade de Economia e Finanças - IBMEC, Rio de Janeiro, 2009.

DAVIS, F. D. Perceived Usefulness, Perceived Ease of Use, and User Acceptance of Information Technology. MIS Quarterly, v. 13, n. 3, pp. 318-340, 1989.

DAVIS, F. D.; BAGOZZI, R. P.; WARSHAW, P. R. User Acceptance of Computer Technology: a compa rison of two theoretical models. Management Science, v. 35, n. 8, pp. 982-1003, 1989.

DODDS, W. B., MONROE, K. B., and GREWAL, D. 1991. "Effects of Price, Brand, and Store Information on Buyers," Journal of Marketing Research (28:3), pp. 307-319.

HOLBROOK, M. B., and HIRSCHMAN, E. C. 1982. "The Experiential Aspects of Consumption: Consumer Fantasies, Feelings, and Fun," Journal of Consumer Research (9:2), pp. 132-140.

KIM, S. S., MALHOTRA, N. K. 2005. “A Longitudinal Model of Continued IS Use: An Integrative View of Four Mechanisms Underlying Post-Adoption Phenomena,” Management Science (51:5), pp. 741-755.

KIM, S. S., MALHOTRA, N. K.,E NARASIMHAN, S. 2005. "Two Competing Perspectives on Automatic Use: A Theoretical and Empirical Comparison,” Information Systems Research (16:4), pp. 418-432.

LIMAYEM, M., HIRT, S. G., and CHEUNG, C. M. K. 2007. "How Habit Limits the Predictive Power of Intentions: The Case of IS Continuance,” MIS Quarterly (31:4), pp. 705-737.

JOHNSON, R. D.; MARAKAS, G. M. Research Report: The Role of Behavioral Modeling in Computer Skills Acquisition: Toward Refinement of the Model. Information Systems Research, v. 11, pp. 402-417, 2000 .

MOORE, G. C.; BENBASAT, I. Development of an instrument to measure the perceptions of adopting an information technology innovation. Information Systems Research, v. 2, n. 3, pp. 192-222, 1991. 
MOORE, G. C. BENBASAT, I. Integrating Diffusion of Innovation and Theory of Reasoned Action Models to Predict Utilization of Information Technology by End-Users. In: KAUTZ, K.; PRIES-HEJE, J. Diffusion and Adaption of Information Technology. Chapman \& Hall, London: pp. 132-146, 1996.

SILVA, J. V. M. ; RODELlO, I. A. . Um Estudo sobre a Aceitação e Uso da Realidade Aumentada em Cenários de Negócio sob a ótica da Teoria Unificada de Aceitação e Uso da Tecnologia. In: Workshop de Realidade Virtual e Aumentada (WRVA 2015), 2015, Presidente Prudente. XII Workshop de Realidade Virtual e Aumentada. São Paulo: Cultura Acadêmica, 2015. v. 1. p. 54-59.

TAYLOR, S., and TODD, P. A. “Assessing IT Usage: The Role of Prior Experience,” MIS Quarterly (19:2), 1995a, pp. 561-570.

TAYLOR, S., and TODD, P. A. "Understanding Information Technology Usage: A Test of Competing Models," Information Systems Research (6:4), 1995b, pp. 144-176.

THOMPSON, R. L., HIGGINS, C. A., and Howell, J. M. "Personal Computing: Toward a Conceptual Model of Utilization,” MIS Quarterly (15:1), 1991, pp. 124-143.

VALLERAND, R. J. 1997. "Toward a Hierarchical Model of Intrinsic and Extrinsic Motivation," in Advances in Experimental Social Psychology, M. Zanna (ed.), New York: Academic Press, pp. 271-360.

VENKATESH, V.; MORRIS, M. G.; DAVIS, G. B.; DAVIS, F. D. User acceptance of Information Technology: toward a unified view. MIS Quarterly, v. 27, n.3, pp. 425-478, 2003.

VENKATESH, V. THONG, J. Y. L.; XU, X. Consumer Acceptance and Use of Information Technology: extending the Unified Theory of Acceptance and Use of Technology. MIS Quarterly, v. 36, n. 1, p. 157-178, Mar., 2012.

\section{Questionário}

Neste questionário, você vai encontrar perguntas sobre sua organização, seu trabalho, e o sistema ERP da sua empresa. É muito importante que você tente responder estas perguntas da forma mais precisa e sincera possível. Não adicione ao questionário qualquer dado pessoal (ex. nome, identidade, etc.) que permita identificálo (a). As informações que você prover são absolutamente confidenciais e serão utilizadas exclusivamente para fins de pesquisa. Muitas das perguntas incluem escalas parecidas com a que está reproduzida abaixo:

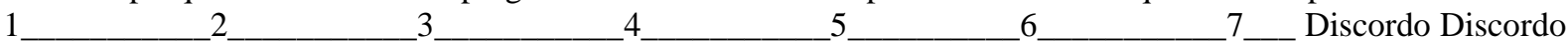
em Discordo Neutro Concordo Concordo em Concordo Inteiramente grande parte parcialmente parcialmente grande parte inteiramente

Ao responder a essas perguntas, indique até que ponto você concorda ou discorda das afirmativas utilizando a escala fornecida.. Ao longo do questionário, várias questões farão referência ao sistema. Em todos os casos estamos nos referindo ao novo sistema ERP sendo implementado ou em uso na sua empresa.

Questionário:

1) Que módulos do sistema ERP você utiliza ou acha que irá utilizar com maior frequiência em seu trabalho?

2) Há quanto tempo você é usuário do sistema ERP? Se ainda não for usuário, por favor, responda o valor 0 (zero). Exemplo: 2 anos e 3 meses.

3) Há quanto tempo você recebeu treinamento no sistema ERP? Se você não tiver recebido treinamento, por favor, responda o valor 0 (zero). Exemplo: 2 meses.

4) Qual a sua idade?

5) Sexo?

( ) Masculino 
( ) Feminino

6) Nível de escolaridade:

( ) Ensino Fundamental

( ) Ensino Médio

( ) Ensino Superior

( ) Pós-Graduação

7) As próximas perguntas devem ser respondidas utilizando escalas similares à que está reproduzida abaixo:

Concordo

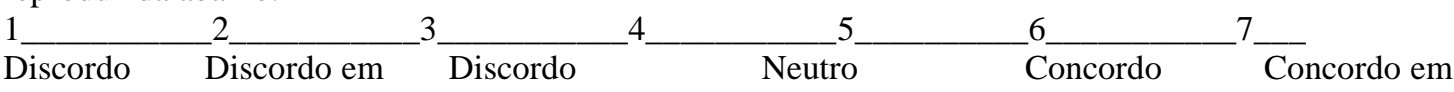
inteiramente

Inteiramente grande parte parcialmente

parcialmente

grande parte

Ao responder, use os números nas escalas para indicar até que ponto você concorda ou

discorda de cada afirmativa.

Nas afirmativas, o termo "sistema" refere-se sempre ao sistema ERP sendo implementado ou

em uso na sua empresa.

Expectativa de Desempenho

PE1. Eu acho o sistema de ERP útil na minha vida diária.

PE2. Usar o sistema de ERP aumenta minhas chances de alcançar as coisas que são importantes para

$\operatorname{mim}$.

PE3. Usar o sistema de ERP me ajuda a realizar as coisas mais rapidamente.

PE4. Usar o sistema aumenta minha produtividade.

Expectativa de Esforço

EE1. Aprender a usar o sistema de ERP é fácil para mim.

EE2. Minha interação com o sistema de ERP é clara e compreensível.

EE3. O sistema de ERP é fácil de usar.

EE4. É fácil, para mim, ser habilidoso no uso do sistema de ERP.

Influência social

SI1. Pessoas que são importantes para mim pensam que eu deveria usar o sistema de ERP.

SI2. As pessoas que influenciam meu comportamento pensam que eu deveria usar o sistema de ERP.

SI3. Pessoas cujas opiniões que eu valorizo preferem usar o sistema de ERP.

Facilitando Condições

FC1. Tenho os recursos necessários para usar o sistema de ERP.

FC2. Tenho o conhecimento necessário para usar o sistema de ERP.

FC3. O sistema de ERP é compatível com outras tecnologias que uso.

FC4. Posso obter ajuda de outros quando tenho dificuldades em usar o sistema de ERP.

Motivação Hedônica

HM1. Usar o sistema de ERP é legal.

HM2. Usar o sistema de ERP é agradável.

HM3. Usar o sistema de ERP é muito divertido.

Valor do preço

PV1. O sistema de ERP é barato.

PV2. O sistema de ERP tem um bom custo-benefício.

PV3. Ao preço atual, o sistema de ERP oferece um bom retorno.

Hábito

HT1. O uso do sistema de ERP tornou-se um hábito para mim.

HT2. Sou viciado em usar o sistema de ERP.

HT3. Eu devo usar o sistema de ERP.

HT4. Usar o sistema de ERP tornou-se natural para mim.

Intenção comportamental

BI1. Pretendo continuar usando o sistema de ERP no futuro.

BI2. Eu sempre tentarei usar o sistema de ERP na minha vida diária. 
BI3. Planejo continuar a usar o sistema de ERP com frequência.

Usar

Escolha sua frequência de uso para cada um dos seguintes:

a) Pesquisar preço.

b) Ver meta.

c) Cotação de venda.

d) Emitir Nota-Fiscal.

e) Gerar boleto de pagamentos.

f) Pesquisar histórico do cliente

g)Cadastrar Cliente

Nota: A frequência variou de "nunca" a "muitas vezes por dia". 